\title{
Singularity: Raychaudhuri Equation once again
}

\author{
Naresh Dadhich \\ Inter-University Centre for Astronomy \& Astrophysics, Post Bag 4, \\ Pune 411 007, India \\ E-mail: nkd@iucaa.ernet.in
}

\begin{abstract}
I first recount Raychaudhuri's deep involvement with the singularity problem in general relativity. I then argue that precisely the same situation has arisen today in loop quantum cosmology as obtained when Raychaudhuri discovered his celebrated equation. We thus need a new analogue of the Raychaudhuri equation in quantum gravity.
\end{abstract}

PACS numbers: 04.20.Jb, 04.2.Cv, 98.80 Dr

\section{Singularity and AKR}

It would not be far from the truth to say that A. K. Raychaudhuri (AKR) had a fascinatingly engaging love affair with the notion of a spacetime singularity at the two ends of his research career. One of his early concerns was to construct a model of a collapsing homogeneous dust ball (he was unaware of Oppenheimer-Snyder collapse) and show that nothing prevented the ball from collapsing down to the centre $r=0$ and thereby demystify the so called Schwarzschild singularity at $r=2 M$ [1]. Then he addressed the most pertinent question of his time: is the cosmic singularity predicted by the FRW model an artifact of the homogeneity and isotropy of space or not? As he explained in his reminiscences [2], inspired by the famous Gödel solution, he was looking for a rotating non-singular solution without closed timelike lines. In the process, he discovered his celebrated equation [3] which made the singularity analysis free of these restrictions. That ultimately led to the powerful Hawking-Pensrose singularity theorems 
[4] which established in a very general setting the inevitability of the occurrence of singularities in Einstein gravity under reasonable energy and causality conditions.

The other end phase began in the mid 1990s. The singularity theorems reigned supreme, particularly since the observation of CMBR [5] had pointed to a singular birth of the Universe in a big bang. Nothing could be happier and more persuasive than observation verifying the prediction of theory. This gave rise to a general belief that singularities were inevitable in general relativity (GR) so long as the dynamics were governed by Einstein's equations and moreover positive energy and causality conditions were respected. However, this belief was shaken by Senovilla's discovery in 1990 of a singularity free cosmological solution [6] which did not violate the energy and causality conditions. How could such a thing happen? It brought forth the main suspect in the proofs of the singularity theorems. Apart from the self-evident assumptions, the theorems also required the existence of a closed trapped surface. This last requirement is certainly not so obvious and self-evident as the other asumptions. That gravity should become so strong in some bound region of space that even light could not escape from it is a very limiting assumption. Indeed, where gravity should become how strong ought to be determined by the field equations rather than by prescription. The said assumption may, however, be reasonable and justifiable for the case of the gravitational collapse of an isolated body. We know from the study of stellar structure that a sufficiently massive body could, after the exhaustion of its nuclear fuel, ultimately undergo indefinite collapse and thereby reach the trapped surface limit. In the case of big bang cosmology what is required is not a trapped surface but instead sufficient amount of matter distribution for the focusing of non-spacelike trajectories at a finite proper time in the past. It is a different matter that the amount required to thermalise the cosmic background radiation is indeed sufficient for the convergence of trajectories in the past [4]. Though this limitation in terms of either a trapped surface or a sufficient amount of energy density was known to experts in the field, it was not talked about much, perhaps in the belief that a singularity free solution would never be found.

In the early 1990s, L. K. Patel, Ramesh Tikekar and myself ob- 
tained some singularity free cosmological solutions [7], in particular one with a stiff fluid equation of state $\rho=p$. During this period, I had several discussions with AKR. We both shared the view that the assumption of the existence of a closed trapped surface almost amounted to putting in a singularity. Nothing could come out of a closed trapped surface nor could the collapse be halted or reversed inside it without violating energy and causality conditions. Thus a singularity would become inevitable. He was also not happy with the genericity condition. In his view it was too complicated and physically not very illuminating. In the ICGC meeting at IUCAA in December 1995, Jose Senovilla and I had discussions with him and he then came out with an insightful comment. He opined that the vanishing of the space average of physical and kinamatic parameters was required for singularity free solutions. We were both struck by this comment which showed a new direction. (See Senovilla's acount in this volume [8]).

AKR had started thinking about singularity free cosmological solutions, but was not yet quite taken up by them. In November 1996, there was an IUCAA sponsored workshop on Inhomogemeous Cosmological Models at North Bengal University, Siliguri. I spoke there on singularity free cosmological solutions. During the talk, AKR asked several probing questions and we had a very lively and engaging discussion. It was indicative of his thought process in trying to understand and resolve intricate and involved conceptual and physical issues. It took him a couple of years before he could start working on the question of the avoidance of the cosmic singularity. This showed his work ethic - deep and long period of contemplation and thought before taking up a problem. This venture took him once again to the question of singularity theorems. He argued that the existence of singularity free cosmological solutions should be recognized and proposed the vanishing of the spacetime averages of all the scalars appearing in the Raychaudhuri equation as a necessary condition for their existence $[9,10,11]$. He later proved a new singularity theorem in which he replaced the occurrence of a closed trapped surface by the non-vanishing of the space averages of all scalars occurring in the Raychaudhuri equation [11]. The vanishing of such space averages was shown to be the key to singularity free cosmological models.

The last paper that AKR wrote was in 2004. In this he attempted 
to deduce the Ruiz-Senovilla family [12] of non-singular solutions for a non-rotating perfect fluid from very general considerations. His procedure was novel, though not mathematically rigorous [13]. A very large family of singularity free cosmological models including some counter examples to his paper [13] had also been found [14]. It is however known that for an imperfect fluid it is easy to construct nonsingular and even oscillating models $[15,16]$. The real challenge is, in fact, in obtaining rotating perfect fluid solutions. Apart from increasing the mathematical complexity, rotation brings in the question of the occurrence of closed timelike lines and consequently causality violation. We have the well-known rotating Gödel universe which has closed timelike lines. Recall that it was precisely the Gödel solution which had set him on the singularity trail. Right at the beginning in the early 1950s, his main aim was to find a rotating fluid solution, hopefully free of any singularity as well as of any closed timelike line. Instead, he discovered his equation. The question remained open and unsolved, however. In fact, AKR returned once again to it at the end. It is undobtedly one of the most challenging open problems in classical gravity today. Ironically, he began and also breathed his last with it on 18 June 2005.

AKR had the profound insight to have identified the key feature of non-singular solutions, namely the vanishing of space averages of physical paramneters. It was the interaction between him and Jose Senovilla which led to the formulation of this conjecture, though each of them had a different perspective on it. AKR's attempt to prove it [11] was not entirely satisfactory and Senovilla has now proved it ultimately [8]. This result should rightly be called the RaychaudhuriSenovilla theorem. It was perhaps the limitation of mathematical and analytical tools that AKR had at his command which came in the way of his proving the theorem rigorously. Yet, had it not been for his insight, the theorem might not have been formulated. Therefore it is to AKR's credit that he showd the right path in understanding singularity free cosmological solutions. If he had the benefit of the right kind of mathematical backup in the mid 1950s, he could conceivably have arrived at the famous singularity theorems. Once again, I believe that the limiting factor was mathematical technology. With utmost reverence and affection, I would like to acknowledge this fact in the true spirit of AKR which embodied academic and itellectual honesty 
and objectivity.

After recording the story of my understanding and perception of the AKR-singularity saga, let me change gears in the next section to argue that his equation in a new avatar is once again badly needed.

\section{Equation once again}

A singularity marks the limiting point of a physical theory. It is enigmatic and calls for a new theory. In Einstein's GR, gravity is nothing but the curvature of spacetime. A gravitational singularity thus means the breakdown of spacetime structure itself and hence the end of everything.

The forces of Newtonian gravity as well as of Maxwell's electric field diverge and are singular at the central location of the mass/charge point. This singularity does not disturb the spacetime background. Rather, it indicates the limit of validity of the theory. For the electric field, we go over to quantum electrodynamics to overcome the classical singularity. For gravity, apart from addressing the singularity, we also need a new theory for the more basic requirement of making it fully universal. So we have Einstein's theory of gravitation, namely GR. But in this new theory, the Newtonian singularity not only persists but in fact attains a more profound all encompassing proportion. One therefore needs a quantum theory of gravity which has to address the question of singularity in the spacetime structure itself.

GR made two profound predictions, one of the black hole and the other of the big bang. Both harboured singularities. In the former case it is hidden behind an event horizon and hence is inaccessible to an external observer. Though the Schwarzschild solution was obtained in 1916 immediately after GR was propounded, its full import as the representation of a static black hole was not realized as late as the late 1960s. A star, collapsing under its own gravity, will go

on collapsing indefinitely upon the exhaustion of all its nuclear fuel and eventually hit the central singularity $r=0$. The latter would be encompassed by a black hole event horizon from which nothing could come out. Penrose in 1969 pronounced that any singularity occurring 
in a gravitational collapse will always be covered by a black hole and this is known as the Cosmic Censorship Conjecture. Oppenheimer and Snyder considered the collapse of a homogeneous dust cloud. Their conclusion is that it collapses down to a singularity covered by a black hole.

In 1924, Friedmann obtained a non-static solution to Einstein's equations representing an expanding model of the universe. In 1929, Hubble's observation of receding galaxies lent observational strength to this model. It was indeed a wonderful marriage of theory and observation. The matter distribution in the universe was assumed to be homogeneous and isotropic. It predicted that the universe, which was now expanding, would have had a singular beginning in a hot big bang when all matter was concentrated within a very small pointlike region.

An important question then arose. Was this singularity an artifact of the symmetries of matter distribution, to wit homogeneity and isotropy, or a generic feature of Einstein's gravity? That is when AKR came on stage and formulated the singularity issue in all its generality and obtained his celebrated equation in 1953 [3]. The equation brought to the fore the new feature that shear as well as pressure contribute positively to gravity, while rotation goes the other way, as expected. Inspired by the Raychaudhuri equation, Penrose, Hawking and Geroch then proved in the mid 1960s their powerful singularity theorems [4] under very general conditions to establish that singularities are inevitable in GR so long as some reasonable energy and causality conditions are satisfied.

Within the classical framework, just as in Maxwell electrodynamics, there is no way to avoid a singularity in Einstein's gravity. That is why one of the main goals of any quantum gravity theory is to address the singularity question. We do not yet have a full-fledged theory of quantum gravity. There are two main attempts. One is string theory which is based on particle physics. The other is the canonical quantization scheme of loop quantum gravity which is based on GR. We shall follow developments in the latter since it directly addresses the singularity issue.

The loop quantum gravity (LQG) idea rests on an important break- 
through achieved by Abhay Ashtekar in 1986. He discovered new variables in which Einstein's equations take a polynomial form [17]. Since then he has spearheaded this approach. This effort is, however, pursued by a comparatively small but highly committed and talented team of researchers [18]. Even in the absence of a full theory, it is instructive and insightful to apply this developing theory to idealized special cases and probe for possible signatures of quantum gravity effects in astrophysical and cosmological observations. Such applications, howsoever tentative, serve as good testbeds for the evolving theory in regard to its right orientation and direction. With that in view, Martin Bojowald and others have, for the past few years, been examining cosmological applications of loop quantum gravity. Such efforts have led to the subject of loop quantum cosmology (LQC) where one considers the symmetry reduced mini superspace and then carries out loop quantum calculations for specific problems of big-bang cosmology, cosmic microwave background radiation and gravitational collapse $[19,20,21,22,23,24]$. The most pertinent question is whether one could have some observational imprint or signature of quantum gravity effects.

Recently, there have been a couple of LQC based calculations to look for quantum effects in astrophysical and cosmological scenarios. The first observable effect of LQC was studied on CMBR in 2003 by Shinji Tsujikawa, Parampreet Singh and Roy Maartens [24]. It turns out that quantum gravity effects could avoid the big bang singularity and there could be a causal passage through it. Its imprint could therefore be seen on the CMBR spectra. In a recent paper, Abhay Ashtekar, T Pawlowski and Parampreet Singh further illuminate on the quantum nature of the big bang [20]. In this context, it is worth recalling one of the first attempts made by $\mathrm{T}$ Padmanabhan and $\mathrm{J}$ V Narlikar in the 1980s towards avoiding the big bang singularity by quantizing conformal degree of freedom [25]. The pertinent question is how to justify in the extreme high energy regime highly restricted degrees of freedom or the reduced mini superspace of LQC? Both considerations suffer from this lack of justification. It should however be noted that, though there is no rigorous derivation of LQC from LQG, the former does have a good theoretical backup with proper caveats. There has also been a consideration of the collapse of a homogeneous scalar field by R Goswami, P Joshi and Parampreet Singh where LQC 
effects make the central singularity evaporate away as radiation [23]. An observational signature of such a quantum evaporation of a naked singularity may be a pulse of intense radiation such as a gamma ray burst (GRB).

We now come back to the old question: is such an avoidance of the big bang or of a collapse singularity an artifact of the symmetry reduced mini superspace or is it generic to LQC?. Near the singularity, curvatures are divergingly high. So it would not be possible to truthfully sustain the assumption of a reduced mini superspace. Once again, we need another AKR today to find a new avatar of his equation. In other words, a new Raychaudhuri equation is urgently required in loop quantum gravity. It may, like the old one, show the way to new general quantum singularity (avoidance) theorems.

It is true that LQC deals with highly restrictive and idealized cases. One should, however, note that such idealized models have the uncanny knack of picking up the physical essence and innate characteristic of real life phenomena. There are several such examples in gravitational theories. The most famous one is, of course, that of the FRW model predicting a big bang singularity and similarly the Oppenheimer-Snyder collapse of a homogeneous dust ball. Despite being a highly idealized case, the model does carry quite truthfully the signature of a general collapse phenomenon. Similar is the case of the Schwarzschild interior solution with a uniform density assumed for the interior of a star. Such an assumption is physically unacceptable since a uniform density would give rise to an infinite sound speed. Nevertheless, the model picks up all the essential features of the stellar interior correctly. It is remarkable that, even when such considerations are highly restricted, idealized and not even entirely physically acceptable, they often correctly indicate general as well as generic features. Such may as well be the case for the highly idealized LQC toy models indicating the quantum avoidance of the big bang and collapse singularities. As already emphasized, what is needed is a Raychaudhuri equation for quantum gravity. It may bring forth some new features like the development of a negative pressure as the singularity is approached. Until then we have to make do with tentative results which may only be indicative of what the full quantum gravity will ultimately establish. 
Finally, all this should be most satisfying and pleasing to one man, Abhay Ashtekar. He made the path breaking discovery two decades back of his famous new variables which set things on track, leading to Loop Quantum Gravity. This idea has now matured sufficiently to make contact with observations. It has indeed been a long and ardous journey. But, at the end of the day, nothing could please one more than to see the clicking of what one had set out do. Admittedly, we are far from a complete theory of quantum gravity and LQG has a long way to go yet. What is important is that it seems to be on the right track [26]. Recent works of Rovelli, Speziale and others [27, 28, 29] on graviton propagators in LQG and of Smolin et al [30] on the origin of the standard model of particle physics from the quantum nature of geometry are indeed very exciting.

\section{Acknowledgement}

I thank Jose Senovilla for recounting and reflecting on our discussion with AKR and also thank Parampreet Singh and Pankaj Joshi for clarifying comments. I also thank my fellow editor of this volume, Probir Roy for reading the manuscript and making changes which have made it more impactful.

\section{References}

[1] A. K. Raychaudhuri, Phys. Rev. 84, 166 (1951).

[2] A. K Raychaudhuri, A Little Reminiscence in Singularities, Black Holes and Cosmic Censorship, ed. P. S. Joshi (IUCAA, 1996).

[3] A. K. Raychaudhuri, Phys. Rev. 90, 1123 (1955).

[4] S. W.Hawking and G. F. R. Ellis, The Large Scale Structure of space-time (Cambridge Univeristy Press, 1973).

[5] A. A. Penzias and R. W. Wilson, Astrophys. J. 142, 419 (1965).

[6] J. M. M. Senovilla, Phys. Rev. Lett. 64, 2219 (1990).

[7] N. Dadhich, L. K. Patel and R Tikekar, Pramana, 44, 303 (1995).

[8] J. M. M. Senovilla, in this volume. 
[9] A. K. Raychaudhuri, Phys. Rev. Lett. 80, 654 (1998).

[10] A. K. Raychaudhuri, A Fresh Look at the Singularity Problem, in The Universe, eds. N. Dadhich and A. K. Kembhavi, (Kluwer, 2000).

[11] A. K. Raychaudhuri, Mod. Phys. Lett. A15, 319 (2000).

[12] E. Ruiz and J. M. M. Senovilla, Phys. Rev. D45, 1995 (1992).

[13] A. K. Raychaudhuri, Gen. Relativ. Grav. 36, 343 (2004).

[14] L. Fernandez-Jambrina and L. M. Gonzalez-Romero, Phys. Rev D66, 024027 (2002).

[15] N. Dadhich, J. Astrophys. Astro. 18, 343 (1997).

[16] N. Dadhich and A. K. Raychaudhuri, Mod. Phys. Lett. A14, 2135 (1999).

[17] A. Ashtekar, Phys. Rev.Lett. 57, 2244 (1986).

[18] A. Ashtekar, Gravity, Geometry and the Quantum, grqc/0605011.

[19] M. Bojowald, Loop, Quantim Cosmology, Living Rev. Rel. 8, 11 (2005), gr-qc/0601085.

[20] A. Ashtekar, T. Pawlowski and P. Singh. Phys. Rev. Lett. 96, 141301 (2006); Phys. Rev. D73, 124038 (2006); D74, 084003 (2006).

[21] A. Ashtekar and M. Bojowald, Class. Qaunt. Grav. 22, 3349 (2005); 23, 391 (2006).

[22] G. Date and G.M. Hossain, Phys. Rev. Lett. 94, 011302 (2005).

[23] R. Goswami, P.S. Joshi and P. Singh, Phys. Rev. Lett. 96, 031302 (2006).

[24] S. Tsujikawa, P. Singh and R. Maartens, Class. Quant. Grav. 21, 5767 (2004).

[25] T. Padmanabhan in Highlights in Gravitation and Cosmology, eds. B. R. Iyer, A. Kembhavi, J. V. Narlikar and C. V. Visheveshwara (Cambridge University Press, 1988), p. 156.

[26] A. Ashtekar, Physics from Geometry, Nature Physics, 2, 725 (2006).

[27] C. Rovelli, Phys. Rev. Lett. 97, 151301 (2006). 
[28] E. Bianchi, L. Modesto, C. Rovelli and S. Speziale, Class. Qaunt. Grav. 23, 6989 (2006).

[29] E. Livine and S. Speziale, JHEP, 0611, 092 (2006).

[30] S. O. Bilson-Thompson, F. Markopoulou and L. Smolin, Quantum Gravity and the Standard Model, hep-th/0603022. 